
\section{International Scientific Journal Theoretical \& Applied Science}

Victor Aleksandrovich Melent'ev

Philosophy Doctor, senior research associate Rzhanov Institute of Semiconductor Physics Siberian Branch of Russian Academy of Sciences (ISP SB RAS) melva@isp.nsc.ru

\author{
p-ISSN: 2308-4944 (print) e-ISSN: 2409-0085 (online) \\ Year: 2016 Issue: 12 Volume: 44 \\ Published: $30.12 .2016 \quad$ http://T-Science.org
}

SECTION 4. Computer science, computer engineering and automation.

\title{
FAULT-TOLERANCE OF HYPERCUBIC AND COMPACT TOPOLOGY OF COMPUTING SYSTEMS
}

Abstract: We research tolerance of the computing systems (CS) to multiple faults. The index of topological fault tolerance is entered, its dependence on girth of graph VS is shown. Comparison of potential parallelism and of fault tolerance of hyper cubic and compact computing systems is this.

Key words: topological fault-tolerance, hypercubic and compact computing systems.

Language: Russian

Citation: Melent'ev VA (2016) FAULT-TOLERANCE OF HYPERCUBIC AND COMPACT TOPOLOGY OF COMPUTING SYSTEMS. ISJ Theoretical \& Applied Science, 12 (44): 98-105.

Soi: http://s-o-i.org/1.1/TAS-12-44-20 Doi: crossef http://dx.doi.org/10.15863/TAS.2016.12.44.20

\section{ОТКАЗОУСТОЙЧИВОСТЬ ГИПЕРКУБИЧЕСКОЙ И КОМПАКТНОЙ ТОПОЛОГИЙ ВЫЧИСЛИТЕЛЬНЫХ СИСТЕМ}

Аннотация: Исследуется устойчивость вычислительных систем (ВС) к множественным отказам. Введен показатель топологической отказоустойчивости, показана его зависимость от обхвата графа ВС. Дано сопоставление потенциального параллелизма и отказоустойчивости гиперкубической и компактной вычислительных систем.

Ключевые слова: топологическая отказоустойчивость, гиперкубические и компактные вычислительные системы.

\section{Работа выполнена при поддержке Российского фонда фундаментальных исследований} (проект №14-07-00169a)

\section{1. Введение}

Эффективное парирование отказов, интенсивность и кратность которых в результате масштабирования вычислительных систем существенно возрастает, заключается в поддержании функциональной и топологической целостности системы и требует адекватной и своевременной реакции на возникающие в связи с отказами ситуации. Для исследования и реализации топологической целостности параллельных систем используют, как правило, две основные модели: модель с полной $k$ отказоустойчивостью подсистем и модель $k$ отказоустойчивых подсистем c амортизацией отказов.

Для первой при возникновении в системе $k$ отказов характерно сохранение ранга $p$ решаемой в подсистеме задачи, и $k$-отказоустойчивость достигается организацией подсистем с минимизированной для заданных $p$ и $k$ избыточностью, достаточной при удалении из графа ВС любых $k$ вершин для изоморфного вложения в этот граф информационного графа задачи ранга $p$. Использование основанных на этой модели подходов в усовершенствовании и сопоставлении топологий вычислительных систем с позиции отказоустойчивости достаточно широко представлено как зарубежными [1, с. 877], [2, с. 565], [3] , так и отечественными исследованиями [4, с. 172], [5]. В качестве основного критерия при этом используют, как правило, максимальный при заданной кратности отказов размер подсистемы, образуемой для решения задач с некоторой вполне определенной информационной топологией, или максимальную при заданном размере подсистемы кратность допускаемых отказов.

ISPC Generalization of scientific results, 
В данной работе проблема топологической отказоустойчивости вычислительных систем рассматривается в контексте модели с амортизацией отказов, согласно которой при возникновении в процессе решения задачи отказов ее ранг $p$ (размер подсистемы) может изменяться до минимально приемлемого значения. При этом, в отличие от обговоренной выше модели, анализ обусловленной топологией отказоустойчивости ВC обычно осуществляют для системной сети связи в целом, не ограничивая ее рамками подсистем, образуемых для решения определенных, обладающих характерными информационными топологиями, задач и используя, как правило, сетевые показатели, статистически усредненные на множестве возможных при заданной кратности отказов конфигураций. Из критериев, имеющих отношение к проблеме отказоустойчивости, используют также критерий синхронизуемости [6], связывающий топологию сети с возможностью бесконфликтного (в пределах заданных временных ограничений, ограничений на приращение трафика и т. п.) общения произвольных вершин.

Отметим, что в силу экспоненциальной сложности точного вычисления большинство таких показателей являются стохастическими. Использование многокритериальной оптимизации в практике построения $\mathrm{BC}$, как правило, еще более усложняет процессы анализа и синтеза обладающих совокупностью заданных свойств топологий при том, что условия совместности/несовместности критериев также не безусловны и обосновываются стохастически. К тому же использование таких показателей в анализе топологической отказоустойчивости крупномасштабных систем не вполне правомерно не только из-за их «комбинаторной взрывоопасности», непропорциональным связанной

(масштабированию системы и увеличению кратности отказов) ростом числа возможных конфигураций.

Во-первых, это связано с тем, что если для задачи существует предел эффективного распараллеливания, то масштабирование системы сверх этого предела не может привести к аналогичному масштабированию ранга этой задачи, в результате чего число задач из решаемого набора, полностью использующих вычислительные (соответственно, и сетевые) ресурсы, с масштабированием системы будет сокращаться.

Во-вторых, что, пожалуй, более существенно - традиционно используемые оценки влияния отказов на изменения потенциала параллелизма вычислительной системы привязаны к изменениям еe сетевых характеристик лишь качественно. Отсутствие формального соответствия значений сетевых показателей $\mathrm{BC}$ ее потенциалу допускает не более чем качественную оценку топологической отказоустойчивости - на уровне «лучше/хуже».

В данной работе предложены показатели топологической отказоустойчивости $\mathrm{BC}$, основанные на предложенной в [7, с. 117-120] модели масштабируемых параллельных вычислений и на сопоставлении топологий по их влиянию на главный критерий качества $\mathrm{BC}-$ на обеспечиваемый ими потенциальный параллелизм. Определена обусловленность топологической отказоустойчивости $\mathrm{BC}$ обхватом ее графа. Установлена формальная взаимосвязь функций топологической отказоустойчивости и масштабируемости. Предложенные показатели апробированы в сравнительной оценке систем с гиперкубической и компактной топологиями.

\section{2. Описание модели масштабируемой вычислительной системы}

Зависимость масштабируемости параллельных систем и решаемых на них задач от топологии оценивается в [7, с. 117-120] на модели масштабируемых параллельных вычислений, разделенной на две составляющие: первая (п. п. 1-4) отнесена к параллельным приложениям и приписывает им свойства неограниченной распараллеливаемости, вторая (п. п. 5-10) характеризует систему, ограничения параллелизма в которой обусловлены дефицитным быстродействием интерконнекта.

Исходные пункты описания этой модели содержат следующие обозначения: $W$ и $w-$ измеряемые временем объемы вычислений при решении произвольной задачи на одном и на $p$ процессорах вычислительной системы; $Q$ и $q-$ измеряемые информационными единицами (байтами) объемы подлежащих обмену данных, соответствующие одному и $p$ задействованным в системе процессорам. Итак,

1. Задача допускает разбиение на произвольное число $p$ информационно связанных параллельных ветвей - $1 \leq p \leq \infty$. Информационный граф распараллеленной на $p$ ветвей задачи может быть нерегулярным, но обязательно связен;

2. Масштабирование данных в задаче с коэффициентом $m$ увеличивает объем вычислений $W$ и объем $Q$ подлежащих обмену данных в $m$ раз;

3. Общий объем вычислений $W$ и объем $Q$ подлежащих обмену данных при разбиении задачи на $p$ параллельных ветвей не зависят от числа процессоров $p$ и распределяются по ним равномерно $-w=W / p$ и $q=Q / p ;$

4. Параллельный алгоритм не содержит скалярных фрагментов; 
5. Все процессоры системы идентичны, их общее число $n$ достаточно для реализации на них $p$ параллельных ветвей; предварительное распределение входных данных по задействованным в параллельном приложении процессорам не требуется;

6. Топология ВС является регулярной ${ }^{1}$ и неполносвязной;

7. Общие объемы $W$ и $Q$ не зависят от топологии сети связи и от используемой $N T$, ограничения на минимальные объемы $w$ и $q$ отсутствуют;

8. Вычислительные и коммуникационные элементы $\mathrm{BC}$ допускают совмещенную во времени работу;

9. Временны́е затраты на обмены пропорциональны расстояниям между информационно смежными в задаче вершинами графа BC;

10. Совокупность используемых в вычислительной системе топологии и NT гарантирует отсутствие сетевых коллизий и связанных с ними задержек.

Такая модель позволила формализовать связь объемов $W$ вычислений и $Q$ обмениваемых данных распараллеливаемой на $p$ ветвей задачи с предельно допускаемыми расстояниями $\partial$ между информационно смежными ее ветвями при заданных значениях ускорения $-S=T_{1} / T_{p}$; здесь $T_{1}$ и $T_{p}$ - времена решения задачи на одном и на $p$ процессорах, соответственно, а $t_{N T}(Q / p)$ известная для используемой в $\mathrm{BC}$ сетевой технологии (далее, NT - Network Technology) функция времени задержки от объема $q=Q / p$ передаваемой информации):

$$
\partial_{S}(p)=\left\lfloor\frac{W}{S \cdot t_{N T}(Q / p)}\right\rfloor ;
$$

Эта формула наглядно демонстрирует зависимость предельных для рассматриваемых в конкретных ВC приложений значений достижимости $\partial$ от быстродействия используемой NT. Она же устанавливает зависимость предельного расстояния между информационно смежными ветвями распараллеливаемой задачи от требуемого ее ускорения и от присущих задаче объемов вычислительных и обменных операций. Таким образом, реализуемые в исследуемой $\mathrm{BC}^{2}$ параллельные приложения могут быть классифицированы по значениям предельных для информационно смежных ветвей расстояний, соответствующих заданному ускорению $S$ при условии топологической адекватности системы этим приложениям.
Определение: Вычислительная система топологически адекватна задаче с присущими ей объемами $W$ вычислительных операций $и$ $Q$ обрабатываемых данных, если топология системь позволяет сконфигурировать подсистему, в которой число прочессоров $p$ u предельное расстояние д между информационно смежными прочессорами соответствуют заданным значениям ускорения $S$ и/или эффективности Е ее решения.

Используемое далее понятие топологической адекватности ВС и реализуемых на ней параллельных приложений базируется на описанной выше модели и формальном ее выражении (1). Если решение $(W, Q)$-задачи обусловлено получаемым для нее ускорением $S$, то число параллельных ветвей $p$ в подсистеме определяет нижнюю границу числа используемых при этом процессоров [7, с. 123].

Понятие изоморфизма в теории графов отражает взаимную однозначность (биективность) отображения вершин сопоставляемых графов одного порядка - при этом каждой вершине одного из них соответствует ровно одна вершина другого, и отношения смежности вершин и их образов совпадают. В теории вычислительных систем проблема изоморфизма состоит в выявлении в графе $G$ системы подграфа, изоморфного информационному графу параллельной задачи $W(p)$ с заданным числом $p$ параллельных ветвей. Имеющая при этом место дискретность в отношении изоморфизма вложения графа $W(p)$ в $G$ (да/нет) не позволяет численно оценивать степень топологической адекватности вычислительной системы и реализуемых ею параллельных приложений. Такую возможность предоставляет введенный в [8, с. 124] показатель масштабируемости $\mu\left(W, G_{\partial}\right)$ системы с $G$ топологией $^{3}$ в реализации на ней задачи с $W$ топологией при определяемом из (1a), (1б) значении достижимости $\partial$ :

$$
\mu_{\partial}(W, G)=n_{\partial}(W, G) / n(G) ;
$$

здесь $\quad n\left(W, G_{\partial}\right) \equiv n_{\partial}(W, G) \quad-\quad$ порядок максимального, изоморфно вкладываемого в граф $\partial$-достижимости системы $G_{\partial}$, подграфа задачи с $W$-топологией.

Равенство единице показателя $\mu_{\partial}(W, G)$, говорит о том, что такой максимально вкладываемый подграф или является суграфом ${ }^{4}$ графа $G_{\partial}$, или изоморфен ему. Понятно, что если $G_{\partial}$-граф полон, то масштабируемость в нем $\partial$ задач с любой топологией максимальна и равна единице, т. е. он адекватен любым Ә-задачам

${ }^{1}$ Степени всех вершин графа ВС одинаковы.

2 Понятие ВC предполагает использование в ней вполне

3 заданной графом $\mathrm{G}$ топологией системы. определенной NT с характерной для нее функиией $t_{N T}(Q / p)$.

${ }^{4}$ Суграф - остовный (вершинно порожденный) подграф.

ISPC Generalization of scientific results, 
независимо от их информационной топологии. Меньшие значения этого показателя для рассматриваемых в системе задач соответствуют меньшей топологической ее адекватности этим задачам.

\section{3. Топологическая отказоустойчивость масштабируемых вычислительных систем}

Как мы уже указывали в описании используемой модели, реализуемые системой параллельные приложения могут быть классифицированы по значениям предельных для информационно смежных ветвей расстояний $\partial$. Подобные ограничения длины пути, связанные с недостаточным для актуальности информационных обменов быстродействием сетевых технологий, имеют место и в других, например, в распределенных информационных системах. Поэтому ценность исследований, отнесенных к сети в целом и игнорирующих потребности актуализации в ней информационных обменов, с увеличением масштаба сетей связи существенно снижается. Традиционное представление их графами, в которых отношения смежности вершин соответствуют физической смежности сетевых узлов, также не способствует исследованию сетей в реализации лимитированных транзитных взаимодействий, доля которых в общем объеме информационных обменов растет непропорционально сетевым масштабам. В предложенных в $[9, \quad$ с. 213] графах $\partial$ достижимости смежность вершин обусловлена заданным предельно допускаемым между ними расстоянием $\partial \geq 1$, что исключает возможность превышения допустимых задержек между информационно смежными ветвями задач.

Для исследования топологических аспектов устойчивости ВС к отказам кратности $l$ в данной работе мы используем графы $\partial(k)$ достижимости, в которых смежность вершин, в отличие от графов Ә-достижимости, лимитирована не только допускаемым расстоянием $\partial$ между ними, но и числом $k=l+1$ не превышающих этого расстояния независимых путей. Отметим при этом, что понятие отказоустойчивости имеет различную окраску при отнесении его к сети связи ВС или к реализующей параллельное приложение подсистеме. В первом случае вполне допустимо использование традиционных сетевых показателей, отнесенных, однако, не только к исходному графу ВС (с $\partial=1)$, а к производным от него графам $\partial(k)$-достижимости (с $\partial>1$, $k \geq 1)$. Если при этом в качестве критерия $\partial(l)$ отказоустойчивости сети связи выбрать, например, связность, то отношение порядка $\partial(k)$ компоненты связности (компоненты связности графа $\partial(k)$-достижимости) к порядку исходного графа ВC даст достаточно наглядное представление о присущем рассматриваемой сети уровне отказоустойчивости. Однако в данной работе нас прежде всего интересуют определяемые топологиями потенциальные возможности отказоустойчивой реализации в ВС параллельных приложений.

\section{4. Граф $\partial(k)$-достижимости}

Определим граф $\partial(k)$-достижимости $G_{\partial(k)}(G)$, он же - граф $k(\partial)$-соединимости $G_{k(\partial)}(G)[9$, с. 239], как надграф графа $G(V, E)$, дополненный ребрами между вершинами $u, v, \in V$ при наличии между ними в графе $G$ не менее $k$ независимых путей с длиной, не превышающей значение заданной достижимости $\partial$. Очевидно, что число $k$ таких путей (соединимость информационно смежных в подсистеме вершин подграфа системы) должно по меньшей мере превышать заданную кратность $l$ отказов: $k \geq l+1$. В этом случае при удалении $l$ каких-либо вершин из соответствующего выполняемой задаче подграфа ВС любые две из оставшихся в нем информационно смежных вершин гарантированно останутся соединенными путем с длиной, не превышающей предельной для этой задачи достижимости $\partial$.

Построить полную проекцию графа $\partial(k)$-достижимости можно сжатием всего одной полной проекции исходного графа. При этом критерием помещения вершины $x$ на $j$-й уровень выстраиваемой проекции будет ее повторяемость в составе $i$-окружений ${ }^{5}$ (при $1 \leq i \leq \partial$ ) вершин $(j-$ 1)-го уровня с кратностью $m_{x}$, превышающей кратность $l$ отказов: $m_{x}>l, m_{x} \geq k$. Вместе с этим должен быть проведен анализ на независимость всех $m_{x} \partial$-путей ${ }^{6}$ между вершинами $(j-1)$-го уровня и порожденными ими вершинами $j$-го уровня сопоставлением промежуточных на каждом пути вершин, при этом пути с непустым множеством совпадений считаются зависимыми. Большее или равное $k$ число выявленных таким образом независимых путей между вершинами $u$ и $v$ в графе $G$ соответствует наличию ребра между ними в графе $G_{\partial(k)}(G)$.

Покажем это на примере 5-мерного $\mathrm{H}_{5}$ гиперкуба. Двоичные коды адресов его вершин для краткости даны здесь в десятичном исчислении. Приведенная ниже одноуровневая проекция $P$ построена для $\partial=3$ из ракурсной вершины 0 , ее 3 -окружение $\mathcal{N}_{3}(0)$ представлено пятью $(s=5)$ 2-подокружениями вершин, смежных ракурсной (это облегчает выявление

\footnotetext{
$5 i$-окружение вершины $b$ в графе $G-$ множество вершин, отстоящих от $x$ на расстоянии $b$.

6 Длина $\partial$-пути, не превышает $\partial$.
} 
независимых $\partial$-путей); выделенная полужирным курсивом первая вершина в каждом из этих подокружений порождает 2-пути в остальные его вершины:

$$
\begin{aligned}
& P=0^{(1,3,2,7,11,19,5,4,7,13,21,9,8,11,13,25,17,16,19,21,25)}, \\
& 0^{(2,3,1,7,11,19,6,4,7,14,22,10,8,11,14,26,18,16,19,20,26)}, \\
& 0^{(4,5,1,7,13,21,6,2,7,14,22,12,8,13,14,28,20,16,21,22,28)}, \\
& 0^{(8,9,1,11,13,25,10,2,11,14,26,12,4,13,14,28,24,16,25,26,28)}, \\
& 0^{(16,17,1,19,21,25,18,2,19,22,26,20,4,21,22,28,24,8,25,26,28)} . \\
& \text { В замкнутом (включаюем вершину } 0)
\end{aligned}
$$
окружении $\mathcal{N}_{3}(0)$, оставим только кратные вершины, индексируя их кратностью, найденной из данной выше проекции $P: \mathcal{N}_{3}(0)=\left\{0,1_{5}, 2_{5}, 3_{2}\right.$, $4_{5}, 5_{2}, 6_{2}, 7_{3}, 8_{5}, 9_{2}, 10_{2}, 11_{3}, 12_{2}, 13_{3}, 14_{3}, 16_{5}, 18_{2}, 19_{3}, 20_{3}$, $\left.21_{3}, 22_{3}, 24_{2}, 25_{3}, 26_{3}, 28_{3}\right\}$. Требование устойчивости к $l$-кратным отказам требует наличия между вершинами $k>l$ независимых путей, поэтому при $l=1 \quad-\quad \mathcal{N}_{3(2)}(0)=\mathcal{N}_{3}(0), \quad$ а $\quad$ при $\quad l=2$ 3(3)-окружение существенно уменьшается: $\mathcal{N}_{3(3)}(0)=\left\{0,1_{5}, 2_{5}, 4_{5}, 7_{3}, 8_{5}, 11_{3}, 13_{3}, 14_{3}, 16_{5}, 19_{3}, 20_{3}, 21\right.$ $\left.{ }_{3}, 22_{3}, 25_{3}, 26_{3}, 28_{3}\right\}$. Далее рассмотрим случай с $\partial=3, k=2(l=1)$.

Заметим, что так как обхват ${ }^{7}$ гиперкуба равен четырем, а заданная достижимость $\partial=2$, то среди вершин из $\mathcal{N}_{3(2)}(0)$ нет таких, расстояние между которыми было бы равным единице ${ }^{8}$. Очевидно также, что если расстояние $d(a-b)$ между вершинами $a$ и $b$ гиперкуба, равное расстоянию $d(A, B)$ между двоичными кодами их адресов, превышает единицу, то число независимых путей между $a$ и $b$ с длиной, равной $d(a-b)$, также больше единицы. Поэтому попарно рассматривая вершины из $\mathcal{N}_{3(2)}(0)$ и исключая вершины с более чем на 3 отличающимися кодами их адресов, получим подмножество вершин, попарно соединенных не менее чем двумя независимыми путями с длиной, не превышающей заданной достижимости $\partial=3$, и порождающих, таким образом, 3(2)-клику $K_{3(2)}$ из девяти вершин:

$$
K_{3(2)}=\{0,1,2,4,8,12,16,20,24\} .
$$

Изменение последовательности рассмотрения вершин из $\mathcal{N}_{3(2)}(0), \quad$ меняя конфигурацию образуемой при этом клики

$$
K_{3(2)}^{\prime}=\{0,2,3,5,6,8,11,13,14\},
$$

оставляет неизменным ее порядок $n\left(K_{3(2)}\right)=n\left(K_{3(2)}^{\prime}\right)=9, \quad$ соответственно, 3(2)-плотность $H_{5}-\varphi_{3(2)}\left(H_{5}\right)=9$.

\section{5. Обхват графа ВС и ее топологическая отказоустойчивость}

7 Обхватом графа называется длина его кратчайшего цикла.

8 Обусловленность минимальной топологической отказоустойчивости ВС обхватом ее графа будет показана в следующем разделе.

ISPC Generalization of scientific results, Scranton, USA
Ограничения отказоустойчивости ВC связаны с обхватом ее графа.

Утверждение: Обхват $g$ графа $G$ вычислительной системы не должен превымать удвоенного для отказоустойчивого решения задачи W значения достижимости $\partial(W)$ :

$$
g(G) \leq 2 \partial
$$

Доказательство. Если обхват $g(G)$ графа ВС превышает удвоенное значение регламентируемой задачей достижимости $\partial$, то в этом графе по определению обхвата не найдется и пары вершин $u, v \in V$, связанных хотя бы двумя независимыми цепями с длинами, не превышающими этой достижимости: $\partial<\lceil g(G) / 2\rceil \Rightarrow k(u, v) \leq 1$. Следовательно, граф $\partial(k)$-достижимости $G_{\partial(k)}$ будет пустым при отличной от нуля кратности $l$ отказов, порядок компоненты связности в нем равен единице, что отрицает возможность конфигурирования отказоустойчивых подсистем с $\partial<\lceil g(G) / 2\rceil$ в принципе.

Понятно, что для набора реализуемых в ВС отказоустойчивой приложений обхват графа ВС обусловлен минимальным в наборе значением достижимости $\partial \min$ :

$$
g(G) \leq 2 \partial_{\min },
$$

Из (2) и из отсутствия кратных ребер между вершинами графа ВС ясно, что для задач, в решении которых из-за дефицитного быстродействия $N T$ не может быть обеспечено большее единицы значение достижимости $\partial>1, \quad$ свойство топологической отказоустойчивости не реализуемо в принципе: даже при использовании полносвязной топологии - $\partial=1 \Rightarrow l=0$. Поэтому для того чтобы, например, гиперкубическая ВС была способна парировать однократные отказы, быстродействие еe $N T$ должно быть, как минимум, достаточным для обеспечения $\partial \geq 2$.

Очевидно, что при кратности $l \geq 1$ отказов $(k \geq 2)$ обусловленное задачами ограничение (2) обхвата графа ВС дополняется ограничением снизу его степени, которая, как и обхват, также влияет на диаметр графа и на его порядок. Это объясняется тем, что в $s$-регулярном графе с заданными значениями порядка $n$ и степени $s$ наибольшей компактностью 9 обладают графы с наибольшими обхватами [10, с. 166], и тем, что число $k$ независимых цепей между двумя вершинами регулярного графа не может превышать его степени $-k \leq s$.

\section{6. Топологическая отказоустойчивость BC}

\footnotetext{
9 Компактные структуры ВC определены как s-регулярные заданного порядка $\mathrm{n}$ графы с минимально возможным при этих $s$ и $n$ диаметром $d$.
} 
В принципе, введенная в [8, с. 138] функция топологической масштабируемости $\mu_{\partial}(G)$ способна дать представление и о топологической отказоустойчивости вычислительной системы. Для этого необходимо функцию плотности $\varphi\left(G_{\partial}\right)$ графа $G$ системы заменить плотностью $\varphi\left(G_{\partial(k)}\right)$ и аргументировать $\varphi\left(G_{\partial(k)}\right)$ не числом $n \equiv n(G)$ узлов в ней (при нулевой кратности отказов $l=0)$, как это делается в $\mu_{\partial}(G)$, а кратностью $l=k-1$ отказов. Изменив при этом обозначение функции топологической масштабируемости с $\mu_{\partial}(G)$ на $\mu_{\partial, l}(n)$, получим:

$$
\mu_{\partial, l}(n)=\varphi\left(G_{\partial(k)}\right) / n(G) .
$$

Давая, однако, количественную характеристику потенциала параллелизма ВС (какая часть общего числа $n(G)$ процессоров может быть использована в $l$-отказоустойчивом решении $\partial$-задач) функция топологической масштабируемости отказоустойчивой ВС (3) дает лишь качественное представление об изменении этого потенциала в сравнении с изначальным (при $l=0)$ потенциалом - $\varphi_{\partial}(G)$, который у топологически различающихся ВС одного порядка, как правило, тоже различен.

Поэтому топологическую $l$-отказоустойчивость $\theta_{n, \partial}(l)$ системы с $G$-топологией $(n \equiv n(G))$ в решении $\partial$-задач определим отношением плотности $\varphi_{\partial(k)}(G) \equiv \varphi\left(G_{\partial(k)}\right) \quad$ графа $\partial(k)$-достижимости $G_{\partial(k)}$, соответствующей определенной для задач из $(1 \mathrm{a}, 6)$ достижимости $\partial$, и заданной кратности $l \geq 1$ отказов $(k=l+1)$, к исключающей наличие отказов $(l=0)$ плотности $\varphi_{\partial}(G) \equiv \varphi\left(G_{\partial}\right)$ графа $\partial$-достижимости $G_{\partial}$ :

$$
\theta_{n, \partial}(l)=\varphi_{\partial(k)}(G) / \varphi_{\partial}(G) .
$$

Сравнивая (3) и (4), нетрудно заметить, что для заданных значений $n, \partial$ и $l$ функция топологической $l$-отказоустойчивости $\theta_{n, \partial}(l)$ равна отношению функции топологической масштабируемости $l$-отказоустойчивой $\mathrm{BC}$ (3) к функции топологической масштабируемости системы $\mu_{\partial}(G)=\varphi_{\partial}(G) / n(G)$, определяемой в предположении отсутствия $(l=0)$ отказов:

$$
\theta_{n, \partial}(l)=\mu_{\partial, l}(n) / \mu_{\partial}(G) .
$$

В отличие от традиционно используемых в сопоставлении ВС с различными топологиями сетевых показателей (диаметра, ширины бисекции), позволяющих судить лишь о качестве (лучше/хуже, больше/меньше) влияния топологии на потенциал распараллеливаемости задач в системе, показатели топологической масштабируемости $\mathrm{BC}-(3)$ и топологической ее отказоустойчивости - (4) позволяют численно оценивать нижнюю (при распараллеливании в системе информационно $\partial$-полносвязных задач) границу такого потенциала при возникновении в процессе их решения отказов кратности $l$. Следует помнить, однако, что такая оценка справедлива лишь при паритетности (идентичности) всех параллельных ветвей задачи.

\section{7. Сопоставление отказоустойчивости ВС с гиперкубической и компактной топологиями}

Итак, в соответствии с используемой моделью параллельных вычислений считаем, что набор выполняемых в ВС параллельных задач классифицирован по значениям допускаемой для них достижимости $\partial$. Область изменения $\partial$ определим интервалом $1<\partial \leq d$, так как для любого связного графа $G$ с обхватом $\lambda(G)>3$ значения плотности $\varphi_{\partial}(G)$ при $\partial=1$ и $\partial \geq d$ известны: $\varphi_{1}(G)=2$ и $\varphi_{d}(G)=n(G)$.

Напомним, что компактные ВС определены в [1, с. 62] как системы, в основе интерконнекта которых лежит топология, заданная регулярным графом $G(V, E)$, диаметр $d\left(G_{n(s)}\right)$ которого при заданных значениях его порядка $n(G)=|V|$ и степени $s(G)$ имеет минимально возможное значение. При $s>2$ условие, связывающее порядок, степень и диаметр $n(s)$-компактного графа, имеет следующий вид:

$$
\frac{s(s-1)^{d-1}-2}{s-2}<n(s) \leq \frac{s(s-1)^{d}-2}{s-2} .
$$

Диаметр $s$-мерного гиперкуба, как известно, $d\left(H_{s}\right)=s$ при том, что его порядок $n\left(H_{s}\right)=2^{s}$. Сравнив диаметры этих графов при одинаковых значениях их порядков и степени

$$
\frac{s(s-1)^{d}-2}{s-2}=2^{s},
$$

получим значение диаметра компактного графа $G_{n(s)}$, предельный порядок которого при той же, что и у гиперкуба $H_{s}$ степени, близок к порядку последнего ${ }^{10}$ :

$$
d\left(G_{n(s)}\right)=[s / \mathrm{lb}(s-1)] .
$$

Заметим, что отношение диаметров $H_{s}$ и $G_{n(s)}$

$$
d\left(H_{s}\right) / d\left(G_{n(s)}\right)=s /[s / \mathrm{lb}(s-1)]=\mathrm{lb}(s-1),
$$

равное двоичному логарифму уменьшенной на единицу степени, указывает на то, что с увеличением степени $s$ разница в диаметрах этих графов значительно возрастает; например, если

10 Особенности рассматриваемых графов не позволяют достигнуть равенства их порядков.
ISPC Generalization of scientific results, 
при $s=5-d\left(H_{5}\right)=5, n\left(H_{5}\right)=32$, и $d\left(G_{26(5)}\right)=2$, $n\left(G_{6}\right)=26, \quad$ то при $s=9$ получим $d\left(H_{5}\right)=9$, $n\left(H_{9}\right)=512$, и $d\left(G_{658(5)}\right)=3, n\left(G_{6}\right)=658$. Понятно, что существенная разница в диаметрах рассматриваемых графов определяет различия в топологической отказоустойчивости основанных на их применении систем.

Выше мы показали, что плотность графа 3-достижимости 5-мерного гиперкуба $H_{5}$ при однократных отказах $-\varphi_{3(2)}\left(H_{5}\right)=9 . \quad$ Из выведенной в [12, с. 23-26] формулы $\partial$-плотности гиперкуба $H_{s}$

$$
n_{\partial}=\left\{\begin{array}{c}
\sum_{i=0}^{\partial / 2}\left(\begin{array}{l}
S \\
i
\end{array}\right) \quad \text { для четных } \partial, \\
\left(\begin{array}{c}
s-1 \\
\lfloor\partial / 2\rfloor
\end{array}\right)+\sum_{i=0}^{\lfloor\partial / 2\rfloor}\left(\begin{array}{l}
S \\
i
\end{array}\right) \text { для нечетных } \partial .
\end{array}\right.
$$

получим $\varphi_{3}\left(H_{5}\right)=10$. Тогда в соответствии с (4) 1-отказоустойчивость гиперкуба $\mathrm{H}_{5}$ $\theta_{32,3}(1)=\varphi_{3(2)}\left(H_{5}\right) / \varphi_{3}\left(H_{5}\right)=0,9$. Из приведенного в разделе 4 статьи 3-окружения $H_{5}$ нетрудно увидеть, что повышение кратности $l$ отказов приводит к уменьшению $3(k)$-плотности этого графа - соответственно уменьшается и его отказоустойчивость: $\varphi_{3(3)}\left(H_{5}\right)=7$ и $\theta_{32,3}(2)=0,7$; $\varphi_{3(4)}\left(H_{5}\right)=5$ и $\theta_{32,3}(3)=0,5$.

Учитывая, что диаметр $d\left(G_{26(5)}\right)$ предельно компактного графа $G_{26(5)}$ равен двум, что его обхват $g\left(G_{26(5)}\right)=2 d\left(G_{26(5)}\right)+1=5, \quad$ что при достижимости $\partial=3$ условие обхвата выполняется и что все фундаментальные циклы этого графа обладают равной обхвату длиной, число $k$ независимых путей при $\partial<d+1-k=1$, а при $\partial \geq 3 \quad k=s$. Поэтому $\forall k \mid 1<k \leq 5$ $3(k)$-плотность $G_{26(5)}-\varphi_{3(k)}\left(G_{26(5)}\right)=26, \quad$ и топологическая отказоустойчивость $\theta_{26,3}(k)=\varphi_{3(k)}\left(G_{26(5)}\right) / \varphi_{3}\left(G_{26(5)}\right)=26 / 26=1$.

То же можно увидеть при рассмотрении графов большей степени. Увеличивающееся при этом отличие в диаметрах гиперкуба и компактного графа не только повышают преимущества последних в построении отказоустойчивых $\mathrm{BC}$, но существенно повышают потенциальный их параллелизм.

\section{8. Заключение}

На базе предложенной в [7] модели масштабируемых параллельных вычислений в работе дано понятие о топологической отказоустойчивости вычислительных систем, предложены показатели оценки влияния топологий на потенциальный параллелизм выполняемых в системе отказоустойчивых приложений. Дано понятие топологической адекватности ВС и решаемых на них задач. Рассмотрены условия топологической отказоустойчивости ВС. Предложенные показатели и методология их определения апробированы на конкретных примерах систем с гиперкубической и компактной топологиями, дан сравнительный анализ обеспечения ими гарантированной отказоустойчивости.

Результаты работы могут быть полезными в сопоставлении и выборе топологий параллельных систем, максимально сохраняющих потенциал распараллеливания задач, требующих отказоустойчивой реализации в условиях множественных отказов.

\section{References:}

1. Hayes JP (1976) graph model for fault-tolerant computing system // IEEE Trans. Comput. 1976. Vol. C.-25. No. 9. p. 875-884.

2. Zimmerman GW, Esfahanian AH (1992) Chordal rings as fault-tolerant loops // Discrete Applied Mathematics. 1992. No. 37/38. p. 563573.

3. (2016) Kitaj planiruet postroit superkompyuter urovnya ehkzaskejla v 2020 godu. Available: http://www.thg.ru/technews/20160503_110013. html/esk tex.pdf (Accessed: 03.06.2016).

4. Karavai MF (1996) Application of the Symmetry Theory in the Analysis and Synthesis of Fault Tolerant Systems //Avtomatika i Telemekhanika. - 1996. - №. 6. - p. 159-173.

5. Donetti L, Hurtado PI, Mufioz MA (2005) Entangled networks, synchronization and optimal network topology // Phys. Rev. Lett. 95 (2005) 188701. Available: https://arxiv.org/pdf/cond-mat/0502230.pdf (Accessed: 20.10.2016).

6. Barahona M, Pecora LM (2002) Synchronization in Small-world Systems // Phys. Rev. Lett. 89, 05410129 July 2002 Available: https://spiral.imperial.ac.uk/bitstream/10044/1/1 


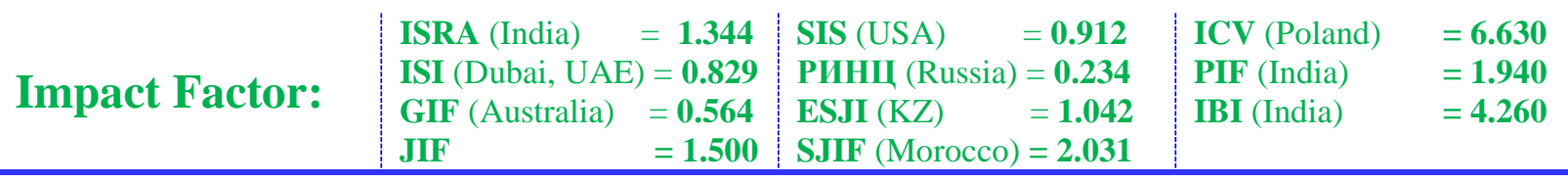

$\underline{0368 / 4 / 0112023 v 1 . p d f}$

(Accessed: 20.10.2016).

7. Melent'ev VA (2015) On topological scalability of computing systems //Upravlenie Bol'shimi Sistemami. - 2015. - T. 58. - p. 115-143.

8. Melent'ev VA, Shubin VI, Zadorozhny AF (2015) Topological scalability of hypercubic parallel systems and tasks. ISJ Theoretical \& Applied Science 11 (31): 122-129.

9. Melent'ev VA (2014) Embedding of subsystems limiting length and number of paths between vertexes of computing system graph, UBS, 47 (2014), 212-246.
10. Melent'ev VA, Shubin VI (2016) On scalability of computing systems with compact topology. ISJ Theoretical \& Applied Science, 11 (43): 164-169.

Doi: http://dx.doi.org/10.15863/TAS.2016.11.43.30.

11. Melent'ev VA (2014) About topological compactness of computing systems. ISJ Theoretical \& Applied Science 11 (19): 59-65.

12. Melentiev VA (2015) Limit configuring of subsystems in hypercubic computing systems // Informacionnye tekhnologii i vychislitelnye sistemy, 2015, No. 2, p. 20-30. 